\title{
Charged black strings and black branes in Lovelock theories
}

\author{
Alex Giacomini, ${ }^{1}$ Marcela Lagos, ${ }^{2}$ Julio Oliva, ${ }^{2}$ and Aldo Vera ${ }^{2}$ \\ ${ }^{1}$ Instituto de Ciencias Físicas y Matemáticas, Universidad Austral de Chile, Casilla 567, Valdivia, Chile \\ ${ }^{2}$ Departamento de Física, Universidad de Concepción, Casilla 160-C, Concepción, Chile
}

(Received 8 March 2018; published 13 August 2018)

\begin{abstract}
It is well known that the Reissner-Norstrom solution of Einstein-Maxwell theory cannot be cylindrically extended to a higher dimension, as with the black hole solutions in vacuum. In this paper we show that this result is circumvented in Lovelock gravity. We prove that the theory containing only the quadratic Lovelock term, the Gauss-Bonnet term, minimally coupled to a $U(1)$ field, admits homogeneous black string and black brane solutions characterized by the mass, charge and volume of the flat directions. We also show that theories containing a single Lovelock term of order $n$ in the Lagrangian coupled to a $(p-1)$-form field admit simple oxidations only when $n$ equals $p$, giving rise to new, exact, charged black branes in higher curvature gravity. For general relativity this stands for a Lagrangian containing the Einstein-Hilbert term coupled to a massless scalar field, and no-hair theorems in this case forbid the existence of black branes. In all these cases the field equations acquire an invariance under a global scaling scale transformation of the metric. As explicit examples we construct new magnetically charged black branes for cubic Lovelock theory coupled to a Kalb-Ramond field in dimensions $(3 m+2)+q$, with $m$ and $q$ integers, and the latter denoting the number of extended flat directions. We also construct dyonic solutions in quartic Lovelock theory in dimension $(4 m+2)+q$.
\end{abstract}

DOI: 10.1103/PhysRevD.98.044019

\section{INTRODUCTION}

In general relativity (GR) in vacuum, black branes are objects that are intrinsic to dimensions higher than four. Since the direct product of Ricci flat manifolds is still Ricci flat, one can construct black branes in GR by a simple oxidation of the black hole solutions of a given dimension $d$ to dimension $D=d+q$ by adding $q$ flat directions. In spite of their simple origin, these spacetimes possess very rich physics. They describe the rapidly rotating limits of the black ring in five dimensions [1] as well as the Myers-Perry black hole in dimension greater than or equal to six [2], since in the latter case the angular momentum is not bounded from above. These rapidly rotating objects inherit the instability of the black branes, the Gregory-Laflamme instability, which is triggered by perturbations traveling along the extended direction with wavelength above a critical value $[3,4]$. Therefore, one can in principle get rid of the instability by the compactification of the extra direction with a radius below the critical wavelength, but then, decreasing the mass of the black hole on the brane, and therefore its radius, the instability reappears. These results

Published by the American Physical Society under the terms of the Creative Commons Attribution 4.0 International license. Further distribution of this work must maintain attribution to the author(s) and the published article's title, journal citation, and DOI. Funded by SCOAP ${ }^{3}$. were extended to charged black strings in low energy string gravity with isometry $\mathbb{R}^{10-d} \times \mathbb{R}_{t} \times S^{d-2}$ in dimension ten, where a dilaton and a $(d-3)$-form [with field strength a $(d-2)$-form] are present. Evidence on the stability of the extremal cases was reported also in [5]. In these solutions it is important to notice that the dilaton cannot be turned off without turning off the $(d-3)$-form field, and therefore, in the case $d=4$ the solution is not continuously connected to an oxidation of the Reissner-Norstrom solution. When the instability takes place, numerical simulations in five dimensions show that the black string may brake into a chain of black holes [6-8], in a finite value of the proper time of an asymptotic observer for generic initial data, providing evidence on a violation of cosmic censorship in asymptotically Kaluza-Klein spacetimes. Recent simulations show a similar behavior for the late time evolution of the black ring instability [9] as well as in the ultraspinning regime of the Myers-Perry solution in dimension six [10], providing evidence on violations of cosmic censorship, for generic initial data as well, in an asymptotically flat spacetime in higher dimensions. For any theory predicting corrections to GR, in particular in the context of string theory, one expects that as the perturbed black string evolves it may unveil regions with high curvature and therefore higher curvature corrections may play a role on the evolution of the spacetime. Consequently, it is natural to look for black brane solutions in the presence of higher curvature terms. 
A good model for exploring the physics of higher curvature gravity are Lovelock theories. These are the most general theories having only the metric as a fundamental field, with second order field equations [11]. Even more, the quadratic Gauss-Bonnet theory appears in the low energy limit of the bosonic and heterotic string theories. The Lagrangian of Lovelock theory is constructed by linearly combining the dimensional continuations of all the Euler densities of even dimensions lower than $D$, each term being a homogeneous polynomial of order $l$ on the Riemann tensor, and is given by

$$
\mathcal{L}=\sqrt{-g} \sum_{l=0}^{\bar{l}} c_{l} \mathcal{L}^{(l)},
$$

with

$$
\mathcal{L}^{(l)}=\frac{1}{2^{l}} \delta_{A_{1} B_{1} \ldots A_{l} B_{l}}^{C_{1} D_{1} \ldots C_{l} D_{l}} R_{C_{1} D_{1}}^{A_{1} B_{1}} \ldots R_{C_{l} D_{l}}^{A_{1} B_{l}}
$$

Here $\bar{l} \leq\left[\frac{D-1}{2}\right]$. The $l=0$ contribution is a bared cosmological term, the $l=1$ is the Einstein-Hilbert Lagrangian and the $l=2$ term is the Gauss-Bonnet term. Explicitly,

$$
\begin{aligned}
& \mathcal{L}^{(0)}=1, \\
& \mathcal{L}^{(1)}=\frac{1}{2} \delta_{A B}^{C D} R_{C D}^{A B}=R, \\
& \mathcal{L}^{(2)}=\frac{1}{4} \delta_{A_{1} B_{1} A_{2} B_{2}}^{C_{1} D_{1} C_{2} D_{2}} R_{C_{1} D_{1}}^{A_{1} B_{1}} R_{C_{2} D_{2}}^{A_{2} B_{2}}=R^{2}-4 R_{A B} R^{A B}+R_{A B C D} R^{A B C D}, \\
& \mathcal{L}^{3}=\frac{1}{8} \delta_{A_{1} B_{1} A_{2} B_{2} A_{3} B_{3}}^{C_{1} D_{1} C_{2} D_{2} C_{3} D_{3}} R_{C_{1} D_{1}}^{A_{1} B_{1}} R_{C_{2} D_{2}}^{A_{2} B_{2}} R_{C_{3} D_{3}}^{A_{3} B_{3}} \\
& =R^{3}-12 R R_{A B} R^{A B}+16 R_{A B} R_{C}^{A} R^{B C}+24 R_{A B} R_{C D} R^{A B C D}+3 R R_{A B C D} R^{A B C D} \\
& -24 R_{A B} R_{C D E}^{A} R^{B C D E}+4 R_{A B C D} R^{A B E F} R_{E F}^{C D}-8 R_{A B C}{ }^{D} R^{A E C F} R^{B}{ }_{E D F} \text {. }
\end{aligned}
$$

This theory shares many of the properties of GR. For generic values of the dimensionful couplings $c_{l}$, the theory admits a Birkhoff's theorem in the sense that spherical symmetry implies the existence of an extra Killing vector which is timelike outside the event horizon of the black hole solutions $[12,13]$. The lapse function of the black hole solution is determined up to a polynomial equation (Wheeler's polynomial) [14-16], and each of the solutions of the polynomial has an asymptotic behavior that matches one of the possible maximally symmetric vacua of the theory $[17,18]$. As in GR one would like to construct black branes, nevertheless, for generic values of the couplings, it is not possible to trivially oxidate the black hole solution of a given dimension $d$ and one has to rely on numerical methods to construct homogeneous black strings. This is done by warping the extended directions with a function of the radial coordinate [19-21], which in the Kaluza-Klein language is read as a dilaton with a nontrivial radial profile. Notwithstanding, when all but one of the constants $c_{l}$ is nonvanishing the theory contains black holes [22,23] (see also [24]), and mimics the behavior of general relativity since in this case the black holes in vacuum can be trivially oxidated [25]. ${ }^{1}$ The black holes on the transverse section

\footnotetext{
${ }^{1}$ This result can be extended to Lovelock theories with a unique maximally symmetric solution provided the black hole on the transverse section is warped by a function of the extended direction $[25,26]$. Also, it has been recently shown that for GR with a negative cosmological constant, homogenous black strings can be constructed at the cost of introducing a massless scalar field which is linear along the extended direction [27].
}

can also be obtained from the general solution with generic couplings in the regime where the radial coordinate is small compared with all the length scales involved in the theory, i.e., $r \ll\left|c_{l} / c_{1}\right|^{\frac{1}{2 l-2}}$ for all $l>1$. This is natural since the highest curvature term must dominate the dynamics in the high curvature regime. Recently it was shown that the black strings constructed in this manner also suffer from a Gregory-Laflamme instability, under spherically symmetric perturbations that travel along the extended directions, in the quadratic and cubic Lovelock theories [28,29], respectively (see also [30] for black branes).

The black hole solutions can be charged under a Maxwell field [22] and it is therefore natural to explore whether these spacetimes may lead to charged black branes. Surprisingly enough, in this paper we show that the solutions of the Gauss-Bonnet theory minimally coupled to a Maxwell field can be trivially oxidated. In GR the equations along the extended directions behave as scalars under the change of coordinates on the brane, and turn out to be incompatible with the trace of the field equations on the brane. Nevertheless, here we show that these equations are indeed compatible if the gravitational theory contains only the Gauss-Bonnet term, i.e., $c_{l}=c_{2} \delta_{(l, 2)}$ in Eq. (1). This leads to new black strings and black branes in Gauss-BonnetMaxwell theory, which is shown in Sec. II. In Sec. III we show an interesting extension of this result by proving that if the action contains a single Lovelock term of order $n$ plus the Maxwell action for a $(p-1)$-form field (with field strength $\left.F_{(p)}\right)$, one can construct homogeneous strings and branes with $F_{(p)} \wedge \star F_{(p)} \neq 0$ only if $n=p$. This implies that the 
solutions of GR coupled to a massless scalar field can be trivially embedded in the same theory in higher dimensions by adding flat directions, nevertheless no-hair theorems imply that no black branes can be constructed in this manner, and that the only static solution with spherical symmetry on the brane is the Janis-Newman-Winicour naked singularity in arbitrary dimension $d$ [31,32], times $\mathbb{R}^{q}$. In Sec. IV we construct new solutions for the cubic Lovelock theory coupled to a three-form field strength, which exploiting the results of Sec. III can be oxidated to describe magnetically charged black branes in dimensions $(3 m+2)+q$, with $m$ and $q$ integers, for which the horizon is given by a product of $m$ three-dimensional spaces of constant curvature $\gamma$ times $\mathbb{R}^{q}$. We also construct new magnetic black brane solutions for the quartic Lovelock theory $\mathcal{R}^{4}$ coupled to a three-form fundamental field, in dimensions $(4 m+2)+q$, as well as dyonic solutions in dimension $(2 m+2)+q$.

\section{NEW CHARGED BLACK STRINGS IN GAUSS-BONNET-MAXWELL THEORY}

Let us first review the obstruction on the construction of black branes in Einstein-Maxwell theory by cylindrical oxidation of the Reissner-Norstrom solution. The field equations for Einstein-Maxwell theory are

$\mathcal{E}^{(1,2)}:=R_{A B}-\frac{1}{2} g_{A B} R-\left(F_{A C} F_{B}^{C}-\frac{1}{4} g_{A B} F_{C D} F^{C D}\right)=0$.

Assuming an electric ansatz of the form

$$
\begin{aligned}
d s^{2} & =d \tilde{s}_{D-q}^{2}+d \vec{x}_{q}^{2} \\
& =-f(r) d t^{2}+\frac{d r^{2}}{g(r)}+r^{2} d \Omega_{D-2-q}^{2}+d \vec{x}_{q}^{2}, \\
A & =A_{t}(r) d t,
\end{aligned}
$$

one obtains two sets of equations:

$$
\tilde{R}_{\mu \nu}-\frac{1}{2} \tilde{g}_{\mu \nu} \tilde{R}=\tilde{F}_{\mu \alpha} \tilde{F}_{\nu}{ }^{\alpha}-\frac{1}{4} \tilde{g}_{\mu \nu} \tilde{F}_{\alpha \beta} \tilde{F}^{\alpha \beta},
$$

and

$$
-\frac{1}{2} g_{x_{i} x_{i}} \tilde{R}=-\frac{1}{4} g_{x_{i} x_{i}} \tilde{F}_{\alpha \beta} \tilde{F}^{\alpha \beta} \quad\left(\text { no sum over } x_{i}\right),
$$

where the Greek indices run along the $d=(D-q)$ dimensional manifold with line element $d \tilde{s}^{2}$, objects with a tilde are intrinsically defined in this manifold and $x_{i}$, with $i=1, \ldots, q$, stand for the Cartesian coordinates along the extended, flat directions. Equation (11) leads to the Reissner-Norstrom solution in $D-q$ dimensions with the gauge potential given by $A_{t}=-Q_{e} / r^{d-3}+A_{\infty}$. Inserting the trace of (11) on Eq. (12) one finds that

$$
(D-q-2) \tilde{F}^{2}=(D-q-4) \tilde{F}^{2},
$$

which is compatible only if the electric charge vanishes, and therefore $A_{t}$ is constant. As expected, this shows that the Reissner-Norstrom solution cannot be extended cylindrically to higher dimensions by just adding flat directions. The factor of the left-hand side of (13) is determined by the fact that

$$
\tilde{g}^{\mu \nu} \tilde{G}_{\mu \nu}=\frac{2-(D-q)}{2} \tilde{R}
$$

For the Gauss-Bonnet theory, whose Lagrangian is given by $\mathcal{L}^{(2)}$ in Eq. (5) the generalized Einstein tensor reads

$$
\begin{aligned}
\mathcal{G}_{A B}^{(2)}= & 2 R R_{A B}-4 R_{A C} R_{B}^{C}-4 R_{C D} R_{A B}^{C D}+2 R_{A C D E} R_{B}^{C D E} \\
& -\frac{1}{2} g_{A B} \mathcal{L}^{(2)},
\end{aligned}
$$

and one can see that in dimension $D$

$$
g^{A B} \mathcal{G}_{A B}^{(2)}=\frac{4-D}{2} \mathcal{L}^{(2)} .
$$

We can couple $\mathcal{L}^{(2)}$ to Maxwell's theory and the field equations then read

$$
\mathcal{E}^{(2,2)}:=\mathcal{G}_{A B}^{(2)}-\left(F_{A C} F_{B}^{C}-\frac{1}{4} g_{A B} F_{C D} F^{C D}\right)=0 .
$$

Splitting the equations using the ansatz of the electrically charged black brane (9) and (10) one obtains

$$
\tilde{\mathcal{G}}_{\mu \nu}^{(2)}=\tilde{F}_{\mu \alpha} \tilde{F}_{\nu}{ }^{\alpha}-\frac{1}{4} \tilde{g}_{\mu \nu} \tilde{F}_{\alpha \beta} \tilde{F}^{\alpha \beta},
$$

and

$-\frac{1}{2} g_{x_{i} x_{i}} \tilde{\mathcal{L}}^{(2)}=-\frac{1}{4} g_{x_{i} x_{i}} \tilde{F}_{\alpha \beta} \tilde{F}^{\alpha \beta} \quad$ (no sum over $\left.x_{i}\right)$.

Now, inserting the trace of the equation defined on the $(D-q)$-dimensional manifold (18) into (19) one obtains

$$
(D-q-4) \tilde{F}^{2}=(D-q-4) \tilde{F}^{2},
$$

which is trivially fulfilled and imposes no extra constraint on the electric field. Therefore, one is left with the system of equations projected on the brane (18), which admits black hole solutions with charge and mass [22]. This shows that it is enough to solve the system (18) which will provide a solution of Gauss-Bonnet-Maxwell theory in $D-q$ dimensions, and admits a cylindrical uplift to dimension 
$D$ with planar extra directions. Explicitly, the following brane spacetime is a solution of the Gauss-Bonnet-Maxwell theory (17):

$$
\begin{aligned}
d s^{2}= & -\left(1-\left(\frac{\mu}{r^{D-q-5}}-\frac{Q^{2}}{r^{2(D-q-4)}}\right)^{\frac{1}{2}}\right) d t^{2} \\
& +\frac{d r^{2}}{1-\left(\frac{\mu}{r^{D-q-5}}-\frac{Q^{2}}{r^{2(D-q-4)}}\right)^{\frac{1}{2}}}+r^{2} d \Omega_{D-q-2}^{2}+d \vec{x}_{q}^{2}, \\
A= & -\sqrt{(D-q-4)(D-q-2)} \frac{Q}{r^{D-q-3}} d t,
\end{aligned}
$$

where $\mu$ and $Q$ are integration constants and $d \Omega_{D-q-2}$ is the line element of a $(D-q-2)$-sphere. This spacetime describes a black brane for $D-q>5$. One can check the consistency with the general argument provided above, and show explicitly that the field equations along the extended directions $\vec{x}$ are trivially fulfilled.

It is interesting to notice that the dynamics of this theory is invariant under global scale transformations of the metric, since under $g_{A B} \rightarrow \xi^{-1} g_{A B}$, the field equations (17) scale as

$$
\mathcal{E}_{A B}^{(2,2)}=0 \rightarrow \xi \mathcal{E}_{A B}^{(2,2)}=0 .
$$

This is not a symmetry of the action but it is a symmetry of the field equations.

\section{LOVELOCK THEORIES WITH A SINGLE TERM AND $p$-FORMS}

The results of the previous section immediately motivate the exploration of Lovelock theories containing a single Lovelock term of order $n$, coupled to a $p$-form field strength, with field equations given by

$$
\begin{aligned}
\mathcal{E}^{(n, p)}:= & \mathcal{G}_{A B}^{(n)}-\left(F_{A C_{1} \ldots C_{p-1}} F_{B}^{C_{1} \ldots C_{p-1}}\right. \\
& \left.-\frac{1}{2 p} g_{A B} F_{C_{1} \ldots C_{p}} F^{C_{1} \ldots C_{p}}\right)=0,
\end{aligned}
$$

and the Maxwell equation for a $(p-1)$-form,

$$
\nabla_{A} F^{A C_{1} \ldots C_{p-1}}=0 .
$$

The term in the parentheses of (24) corresponds to the energy-momentum tensor of a $(p-1)$-form field with field strength $F_{(p)}$, and $\mathcal{G}_{A B}^{(n)}$ is the Lovelock tensor of order $n$, i.e.,

$$
\mathcal{G}_{B}^{A(n)}:=-\frac{1}{2^{n+1}} \delta_{B D_{1} \ldots D_{2 n}}^{A C_{1} \ldots C_{2 n}} R_{C_{1} C_{2}}^{D_{1} D_{2}} \ldots R_{C_{2 n-1} C_{2 n}}^{D_{2 n-1} D_{2 n}},
$$

which reduces to the Einstein tensor when $n=1$. This tensor is obtained by the Euler-Lagrange derivative with respect to the metric of the integral spacetime integral of $\sqrt{-g} \mathcal{L}^{(n)}$ with $\mathcal{L}^{(n)}$ defined in (2), and it is therefore symmetric and divergenceless. It is important to notice the following identity:

$$
g^{A B} \mathcal{G}_{A B}^{(n)}=\left(\frac{2 n-D}{2}\right) \mathcal{L}^{(n)} .
$$

We are interested in homogenous black strings and branes with metrics of the form (9), and therefore, as in the electromagnetic case, let us assume that the fundamental $(p-1)$-form field depends only on the coordinates on the brane. This requirement is general enough also to admit solutions with magnetic charges. We can now project the equations of the Lovelock theory supported by a $(p-1)$-form (24) on the brane and along the $q$ extended directions, which respectively lead to

$\tilde{\mathcal{G}}_{\mu \nu}^{(n)}=\left(\tilde{F}_{\mu \alpha_{1} \ldots \alpha_{p-1}} \tilde{F}_{\nu}^{\alpha_{1} \ldots \alpha_{p-1}}-\frac{1}{2 p} \tilde{g}_{\mu \nu} \tilde{F}_{\alpha_{1} \ldots \alpha_{p}} \tilde{F}^{\alpha_{1} \ldots \alpha_{p}}\right)$,

$-\frac{1}{2} g_{x_{i} x_{i}} \tilde{\mathcal{L}}^{(n)}=-\frac{1}{2 p} g_{x_{i} x_{i}} \tilde{F}_{\alpha_{1} \ldots \alpha_{p}} \tilde{F}^{\alpha_{1} \ldots \alpha_{p}} \quad$ (no sum over $x_{i}$ ).

Inserting (29) on the trace of the $(D-q)$-dimensional equations (28), one obtains

$$
(D-q-2 n) \tilde{F}_{\alpha_{1} \ldots \alpha_{p}} \tilde{F}^{\alpha_{1} \ldots \alpha_{p}}=(D-q-2 p) \tilde{F}_{\alpha_{1} \ldots \alpha_{p}} \tilde{F}^{\alpha_{1} \ldots \alpha_{p}},
$$

which for $\tilde{F}_{\alpha_{1} \ldots \alpha_{p}} \tilde{F}^{\alpha_{1} \ldots \alpha_{p}} \neq 0$ is consistent only if $n=p$. This shows that we can cylindrically uplift the solutions of Lovelock theory with a single Lovelock term of order $n$, only when they are supported by an $(n-1)$-form field. It is therefore enough to solve the equations on the brane (28) to find new extended black objects. The field equations of such theory also acquire a global scaling symmetry since under $g_{A B} \rightarrow \xi^{-1} g_{A B}$, one has

$$
\mathcal{E}_{A B}^{(n, n)}=0 \rightarrow \xi^{n-1} \mathcal{E}_{A B}^{(n, n)}=0 .
$$

Note that in order to have a nonvanishing contribution of the Lovelock theory of order $n$ to the field equations we need $d>2 n$.

It is interesting to notice that Einstein theory supported by a single massless scalar field belongs to the special class of theories singled out by the previous arguments. Indeed in such a case the equations on the brane read

$$
\tilde{R}_{\mu \nu}-\frac{1}{2} \tilde{g}_{\mu \nu} \tilde{R}=\partial_{\mu} \phi \partial_{\nu} \phi-\frac{1}{2} \tilde{g}_{\mu \nu} \tilde{g}^{\alpha \beta} \partial_{\alpha} \phi \partial_{\beta} \phi,
$$

while the equations along the extended directions reduce to 


$$
\tilde{R}=\tilde{g}^{\alpha \beta} \partial_{\alpha} \phi \partial_{\beta} \phi,
$$

which is trivially implied by the trace of (32). Due to no-hair theorems, it is not possible to obtain cylindrically extended black objects in this system, nevertheless for spherical symmetry on the brane, this shows that one can indeed cylindrically extend the singular JanisNewman-Winicour [31] solution from four dimensions to $D=(4+q)$-dimensions as a solution of the EinsteinKlein-Gordon system in dimension $D$. This can be extended to arbitrary $d$ by considering the ZanniasXanthopoulos singular solution [32].

We have seen in the previous section that for the GaussBonnet theory coupled to a Maxwell field one can indeed construct black branes. In the following section we construct new solutions for cubic and quartic Lovelock theories supported by a three-form and four-form field strength, respectively, describing black strings and branes.

\section{NEW CHARGED BLACK P-BRANES IN LOVELOCK GRAVITY}

In the previous section we have shown that the Lovelock theory of order $n$ coupled to a $(p-1)$-form with $p=n$ admits black brane solutions, provided one can find a black object on the brane. In this section we construct new solutions in this class for cubic and quartic Lovelock theory coupled to a two-form and a three-form, respectively.

\section{A. Cubic Lovelock theory and a Kalb-Ramond field}

Let us propose the following ansatz for the metric:

$$
\begin{aligned}
d s^{2} & =d \tilde{s}_{d}^{2}+d \vec{x}_{q}^{2} \\
& =-f(r) d t^{2}+\frac{d r^{2}}{f(r)}+r^{2}\left(d \Omega_{(1)}^{2}+\cdots+d \Omega_{(m)}^{2}\right)+d \vec{x}_{q}^{2}
\end{aligned}
$$

where $d \Omega_{(i)}$ stands for the line element of the $i$ th threedimensional, constant curvature manifold of curvature $\gamma$, normalized to \pm 1 (it can be checked that the case $\gamma=0$ does not lead to black hole solutions). For the sake of concreteness one could use the following coordinate chart $\left(x_{i}, y_{i}, z_{i}\right)$ on the $i$ th three-dimensional manifold with line element $d \Omega_{(i)}$ such that

$d \Omega_{(i)}^{2}=\frac{d x_{i}^{2}+d y_{i}^{2}+d z_{i}^{2}}{\left(1+\frac{\gamma}{4}\left(x_{i}^{2}+y_{i}^{2}+z_{i}^{2}\right)\right)^{2}} \quad($ no sum over $i)$,

and the following, natural, magnetic ansatz for the threeform field strength

$$
F_{(3)}=P \sum_{i=1}^{m} \operatorname{Vol}\left(\Omega_{(i)}\right),
$$

where $P$ is a constant and which in components reads

$$
\begin{aligned}
F_{\mu \nu \lambda}^{(3)}= & \frac{P}{\left(1+\frac{\gamma}{4}\left(x_{1}^{2}+y_{1}^{2}+z_{1}^{2}\right)\right)^{3}} \delta_{[\mu}^{x_{1}} \delta_{\nu}^{y_{1}} \delta_{\lambda]}^{z_{1}}+\cdots \\
& +\frac{P}{\left(1+\frac{\gamma}{4}\left(x_{m}^{2}+y_{m}^{2}+z_{m}^{2}\right)\right)^{3}} \delta_{[\mu}^{x_{m}} \delta_{\nu}^{y_{m}} \delta_{\lambda]}^{z_{m}}
\end{aligned}
$$

(no sum over $m$ ).

The dimension of the spacetime is therefore $D=d+$ $q=(2+3 m)+q$.

In this manner, as usual, the equations for the two-form are fulfilled:

$$
\nabla_{\mu} F_{(3)}^{\mu \nu \lambda}=0
$$

without any constraint on the magnetic charge $P$. One therefore has to deal with the generalized Einstein equations,

$\mathcal{E}_{A B}^{(3,3)}:=c_{3} \mathcal{G}_{A B}^{(3)}-\left(F_{A C D} F_{B}^{C D}-\frac{1}{6} g_{A B} F_{C D E} F^{C D E}\right)=0$,

and then solve the lapse function $f(r)$ from the system,

$$
\begin{aligned}
&- \frac{c_{3}}{2^{4}} \delta_{B D_{1} \ldots D_{6}}^{A C_{1} \ldots C_{6}} R_{C_{1} C_{2}}^{D_{1} D_{2}} R_{C_{3} C_{4}}^{D_{3} D_{4}} R_{C_{5} C_{6}}^{D_{5} D_{6}} \\
&-\left(F_{C D}^{A} F_{B}^{C D}-\frac{1}{6} \delta_{B}^{A} F_{C D E} F^{C D E}\right)=0
\end{aligned}
$$

Note that we have explicitly included the coupling ${ }^{2} c_{3}$.

With this ansatz, one can use the results from the previous sections to show that the equations $\mathcal{E}_{x_{i} x_{i}}^{(3,3)}=0$ (no sum over $x_{i}$ ) are implied by the trace of the equations on the brane. Out of these equations one can show that

$$
\mathcal{E}_{t}^{(3,3) t}=\mathcal{E}_{r}^{(3,3) r}
$$

The equations projected along the angles $\Omega_{(i)}$ are all equal and reduce to a linear combination of the $\mathcal{E}_{t}^{(3,3) t}$ equation and its derivative with respect to the radial coordinate, as expected from diffeomorphism invariance. Consequently, one is left with a single master equation that admits a first integral and leads to the following Wheeler's-like polynomial for $f=f(r)$ :

\footnotetext{
${ }^{2}$ For some explicit examples of solutions including charged black holes with planar horizons, Taub-Nut black holes and their coupling with nonlinear electrodynamics in general Lovelock theories containing the cubic term see the works in Ref. [33].
} 


$$
\begin{gathered}
f^{3}-\frac{6 \gamma}{(d-3)} f^{2}+\frac{12}{(d-4)(d-3)} f+\frac{1}{54} \frac{(d-8) ! P^{2}}{(d-3) ! c_{3}} \\
+\frac{\mu}{r^{d-7} c_{3}}-\frac{8 \gamma(d-8)}{(d-6)(d-7)(d-3)(d-4)}=0 .
\end{gathered}
$$

Here $\mu$ is an integration constant.

Assuming the existence of a horizon located at $r=r_{+}>0$, we can compute the temperature and the entropy density of the black string [34], which respectively read

$$
\begin{aligned}
& T=\frac{f^{\prime}\left(r_{+}\right)}{4 \pi}=\frac{1}{48} \frac{(d-7)(d-4)(d-3) \mu}{c_{3} \pi r_{+}^{d-6}}, \\
& s=64 \pi^{2} c_{3}(d-5)(d-2) r_{+}^{d-6},
\end{aligned}
$$

and $\mu=\mu\left(r_{+}\right)$is given by

$\mu\left(r_{+}\right)=\frac{(d-2) !(d-5)}{(d-7) !}\left(8 \gamma c_{3}(d-8)-\frac{1}{54} \frac{P^{2}}{(d-5)}\right) r_{+}^{d-7}$

As expected, the positivity of the temperature and entropy imply $c_{3}>0$ and $\mu>0$, which by virtue of (46) and for $d>8$ imply $\gamma=1$ (the case $d=8$ does not lead to black holes). Therefore, the horizon of the black branes is given by the product of $m$ three-spheres times $\mathbb{R}^{q}$. Figure 1 depicts the lapse function for some values of the integration constants in dimensions $d=11$ and $d=14$.

The solution with vanishing integration constants, $\mu=P=0$, has the form

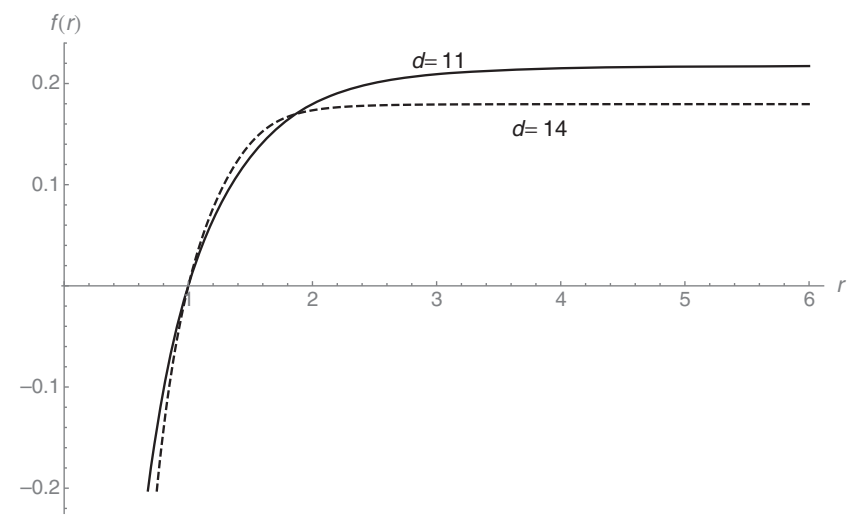

FIG. 1. Charged black holes for cubic Lovelock theory supported by a three-form field strength. $f(r)$ for $c_{3}=1, r_{+}=1$ and magnetic charge $P=1$, for dimensions $d=11$ and $d=14$. The event horizon is a product of three-spheres and this solution can be trivially oxidated to higher dimensions by adding a factor $\mathbb{R}^{q}$ to the metric.

$$
\begin{aligned}
d s_{0}^{2}= & -\alpha_{d}(P=0, \mu=0) d t^{2}+\frac{d r^{2}}{\alpha_{d}(P=0, \mu=0)} \\
& +r^{2}\left(d \Omega_{(1)}^{2}+\cdots+d \Omega_{(m)}^{2}\right)+d \vec{x}_{q}^{2},
\end{aligned}
$$

with $\alpha_{d}$ a real solution of the cubic polynomial (43). This spacetime has a curvature singularity at the origin that, as shown in Fig. 1, can be covered by an event horizon for nonvanishing values of the integration constants. The situation is similar to what occurs for Lifsthiz spacetimes, where the Lifshitz background is singular at the origin (it contains timelike incomplete geodesics), but this singularity is hidden by an event horizon in the case of Lifshitz black holes (see e.g., [35]). This behavior is also reminiscent of gravitating monopoles [36].

The asymptotic behavior of the lapse function is

$$
f(r)=\alpha_{d}(P)-\frac{\tilde{\mu}(d, \mu, P)}{r^{\frac{d-7}{3}}}+\cdots,
$$

where $\cdots$ denotes subleading terms. Here $\alpha_{d}$ and $\tilde{\mu}$ depend on the dimension as well as on the integration constants $\mu$ and $P$. It is interesting to notice that the first dependence in $r$ has the usual falloff for the black hole solution of the cubic Lovelock theory in vacuum [22], therefore one may expect that for a fixed $P=\bar{P}$, these solutions should have a finite mass when the energy density is measured with respect to the background:

$$
\begin{aligned}
d s_{\infty}^{2}= & -\alpha_{d}(\bar{P}) d t^{2}+\frac{d r^{2}}{\alpha_{d}(\bar{P})} \\
& +r^{2}\left(d \Omega_{(1)}^{2}+\cdots+d \Omega_{(m)}^{2}\right)+d \vec{x}_{q}^{2},
\end{aligned}
$$

with a nonvanishing magnetic flux. It is interesting to notice also that the term that decays as $r^{-(d-7) / 3}$ has a slower falloff than that of an asymptotically flat solution with finite mass in general relativity $\left(r^{-(d-3)}\right)$, nevertheless since only the cubic term is present, the mass will come from the term with falloff $r^{-(d-7) / 3}$ on the lapse function.

\section{B. Quartic Lovelock theory and a three-form: Magnetically charged solutions}

Let us now construct new black brane solutions of quartic Lovelock theory coupled to a four-form field strength. We will first construct solutions with magnetic charge only, for which we use the ansatz (34) and (35), where now $d \Omega_{(i)}$ stands for the line element of the $i$ th fourdimensional, constant curvature manifold of curvature $\gamma$, normalized to \pm 1 . Again for concreteness a coordinate chart $\left(x_{i}, y_{i}, z_{i}, w_{i}\right)$ on the $i$ th four-dimensional manifold with line element $d \Omega_{(i)}$ can be used and then

$$
d \Omega_{(i)}^{2}=\frac{d x_{i}^{2}+d y_{i}^{2}+d z_{i}^{2}+d w_{i}^{2}}{\left(1+\frac{\gamma}{4}\left(x_{i}^{2}+y_{i}^{2}+z_{i}^{2}+w_{i}^{2}\right)\right)^{2}} \quad(\text { no sum over } i),
$$


and now the magnetic ansatz for the four-form reads

$$
F_{(4)}=P \sum_{i=1}^{m} \operatorname{Vol}\left(\Omega_{(i)}\right),
$$

which in components turn out to be

$$
\begin{aligned}
F_{\mu \nu \lambda \rho}^{(4)}= & \frac{P}{\left(1+\frac{\gamma}{4}\left(x_{1}^{2}+y_{1}^{2}+z_{1}^{2}+w_{1}^{2}\right)\right)^{3}} \delta_{[\mu}^{x_{1}} \delta_{\nu}^{y_{1}} \delta_{\lambda}^{z_{1}} \delta_{\rho]}^{w_{1}}+\cdots \\
& +\frac{P}{\left(1+\frac{\gamma}{4}\left(x_{m}^{2}+y_{m}^{2}+z_{m}^{2}+w_{m}^{2}\right)\right)^{3}} \delta_{[\mu}^{x_{m}} \delta_{\nu}^{y_{m}} \delta_{\lambda}^{z_{m}} \delta_{\rho]}^{w_{1}},
\end{aligned}
$$

with no sum over $m$. Now the spacetime dimension is $D=d+q=(2+4 m)+q$. Again, Maxwell equations for the fundamental three-form are trivially fulfilled and introducing the gravitational coupling $c_{4}$ one is left with the system

$$
\begin{aligned}
\mathcal{E}_{A B}^{(4,4)} & :=c_{4} \mathcal{G}_{A B}^{(4)}-\left(F_{A C D E} F_{B}^{C D E}-\frac{1}{8} g_{A B} F_{C D E H} F^{C D E H}\right) \\
& =0
\end{aligned}
$$

which explicitly reads

$$
\begin{aligned}
&- \frac{c_{4}}{2^{5}} \delta_{B D_{1} \ldots D_{8}}^{A C_{1} \ldots C_{8}} R_{C_{1} C_{2}}^{D_{1} D_{2}} R_{C_{3} C_{4}}^{D_{3} D_{4}} R_{C_{5} C_{6}}^{D_{5} D_{6}} R_{C_{7} C_{8}}^{D_{7} D_{8}} \\
&-\left(F_{C D E}^{A} F_{B}^{C D E}-\frac{1}{8} g_{A B} F_{C D E H} F^{C D E H}\right)=0 .
\end{aligned}
$$

As before, since there is a single metric function and the system comes from an action that is invariant under diffeomorphisms, it is enough to integrate the $(t t)$ equation that leads to the following Wheeler's-like quartic polynomial:

$$
\begin{aligned}
f^{4} & -\frac{12 \gamma}{(d-3)} f^{3}+\frac{18(3 d-16) \gamma^{2}}{(d-5)(d-4)(d-3)} f^{2}-\frac{108 \gamma(d-8)}{(d-7)(d-5)(d-4)(d-3)} f \\
& -\frac{1}{384} \frac{(d-10) ! P^{2}}{(d-3) ! c_{4}}+\frac{27\left(3 d^{2}-48 d+188\right)}{(d-9)(d-8)(d-7)(d-5)(d-4)(d-3)}-\frac{\mu}{r^{d-9} c_{4}}=0 .
\end{aligned}
$$

Again, $\mu$ is an integration constant. The expression for the temperature and entropy density of a black hole with horizon located at $r=r_{+}$read

$T=\frac{f^{\prime}\left(r_{+}\right)}{4 \pi}=\frac{1}{432} \frac{\mu(d-9)(d-7)(d-5)(d-4)(d-3)}{(d-8) \gamma \pi c_{4} r_{+}^{d-8}}$,

$s=216 \pi^{3} \gamma(d-8)(d-6)(d-2) c_{4} r_{+}^{d-8}$,

and in this case $\mu=\mu\left(r_{+}\right)$is given by

$$
\begin{aligned}
\mu\left(r_{+}\right)= & \frac{(d-10) !}{(d-3) !}\left[27\left(3 d^{2}-48 d+188\right)(d-6) c_{4}\right. \\
& \left.-\frac{1}{384} P^{2}\right] r_{+}^{d-9} .
\end{aligned}
$$

Considering the latter expression plus restricting the entropy and temperature to be positive, implies $c_{4}>0$, $\mu>0$ and $\gamma=1$. Consequently, in this case the event horizon of the black branes has the local geometry of the product of $m$ four-spheres times $\mathbb{R}^{q}$. In Fig. 2 we show some plots of the lapse functions for different values of the integration constants and dimensions.

As before, when $r \rightarrow+\infty$, the lapse function approaches a constant $\alpha_{d}(P)$ plus a term proportional to a function $\tilde{\mu}(d, \mu, P)$ that decays as $r^{-(d-9) / 3}$. This coincides with the asymptotic falloff of the solution in quartic Lovelock theory in vacuum [22].

\section{Quartic Lovelock theory and a three-form: Dyonic solutions}

Finally, let us construct new black brane solutions with both electric and magnetic charge, i.e., dyonic solutions. We assume that the line element is given by (34) and (35) and now $d \Omega_{(i)}$ stands for the line element of the $i$ th twodimensional manifold of constant curvature $\gamma$, normalized to \pm 1 . One can choose the coordinate patch $\left(x_{i}, y_{i}\right)$ on the $i$ th two-dimensional manifold such that

$$
d \Omega_{(i)}^{2}=\frac{d x_{i}^{2}+d y_{i}^{2}}{\left(1+\frac{\gamma}{4}\left(x_{i}^{2}+y_{i}^{2}\right)\right)^{2}} \quad(\text { no sum over } i) .
$$

In order to turn on the electric and magnetic parts of the field strength we assume

$$
\begin{aligned}
F_{(4)}= & Q d t \wedge d r \wedge \sum_{i} \operatorname{Vol}\left(\Omega_{(i)}\right) \\
& +P \sum_{i<j} \operatorname{Vol}\left(\Omega_{(i)}\right) \wedge \operatorname{Vol}\left(\Omega_{(j)}\right),
\end{aligned}
$$

which in components reads 


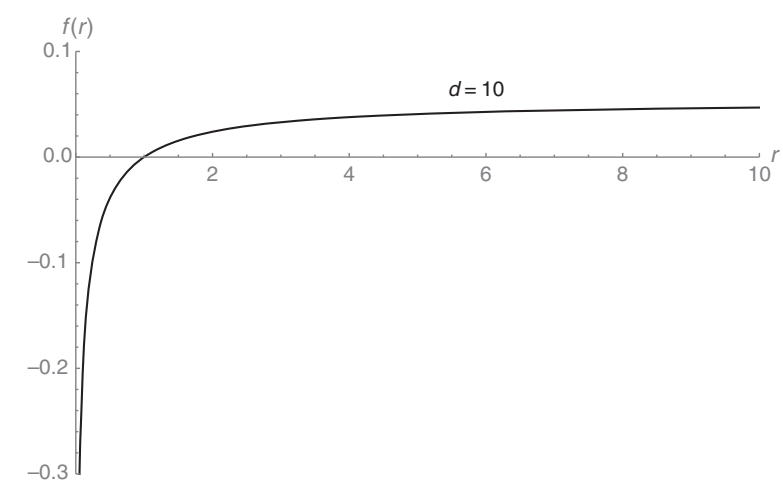

(a)

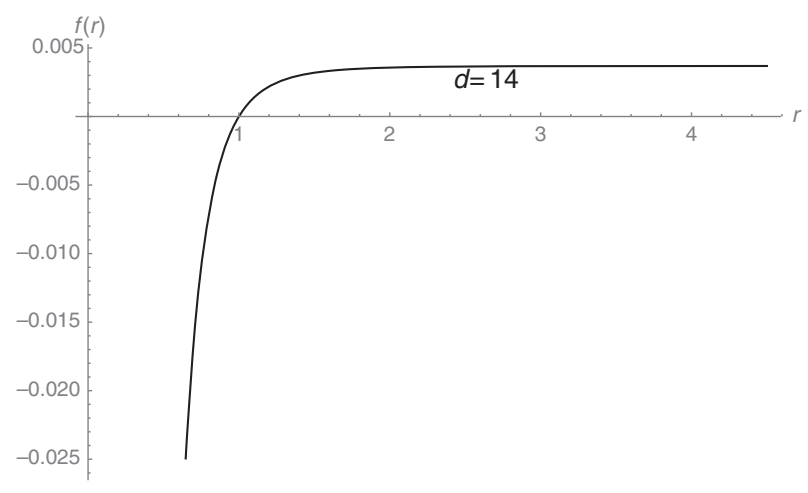

(b)

FIG. 2. Magnetically charged black holes for quartic theory supported by a four-form field strength in dimensions $d=10$ (left panel with $P=2900$ ) and $d=14$ (right panel with $P=550$ ). In both figures we have chosen $c_{4}=1$ and $r_{+}=1$. The event horizon is given by the product of four-spheres and these solutions can be cylindrically oxidated to higher dimensions by adding a factor $\mathbb{R}^{q}$ to the metric.

$$
F_{\mu \nu \lambda \rho}^{(4)}=P \sum_{i<j}^{m} \frac{\delta_{[\mu}^{x_{i}} \delta_{\nu}^{y_{i}} \delta_{\lambda}^{x_{j}} \delta_{\rho]}^{y_{j}}}{\left(1+\frac{\gamma}{4}\left(x_{i}^{2}+y_{i}^{2}\right)\right)\left(1+\frac{\gamma}{4}\left(x_{j}^{2}+y_{j}^{2}\right)\right)}+Q \sum_{i}^{m} \frac{\delta_{[\mu}^{t} \delta_{\nu}^{r} \delta_{\lambda}^{x_{i}} \delta_{\rho]}^{y_{i}}}{\left(1+\frac{\gamma}{4}\left(x_{i}^{2}+y_{i}^{2}\right)\right)}
$$

The spacetime dimension is now $D=d+q=(2+2 m)+q$. Maxwell equations for the fundamental three-form are fulfilled identically and as before the system (54) leads to a single polynomial equation that resembles Wheeler's polynomial,

$$
\begin{aligned}
f^{4} & -\frac{4 \gamma}{(d-3)} f^{3}+\frac{6 \gamma^{2}}{(d-5)(d-3)} f^{2}-\frac{4 \gamma}{(d-7)(d-5)(d-3)} f-\frac{\mu}{r^{d-9} c_{4}} \\
& -\frac{1}{768} \frac{(d-10) !(d-4) P^{2}}{c_{4}(d-3) !}+\frac{1}{192} \frac{(d-9) ! Q^{2}}{c_{4}(d-7)(d-3) ! r^{2(d-8)}}+\frac{\gamma^{2}}{(d-9)(d-7)(d-5)(d-3)}=0 .
\end{aligned}
$$

Temperature and entropy density read

$$
\begin{aligned}
& T=\frac{f^{\prime}\left(r_{+}\right)}{4 \pi}=\frac{(d-5)(d-3)}{16 \gamma \pi c_{4} r_{+}^{d-8}}\left[\mu(d-9)(d-7)-\frac{1}{96} \frac{(d-9) !(d-8)}{(d-3) !} \frac{Q^{2}}{r_{+}^{d-7}}\right], \\
& s=16 \pi^{2} \gamma c_{4}(d-6)(d-4)(d-2) r_{+}^{d-8}
\end{aligned}
$$

and $\mu=\mu\left(r_{+}\right)$in this case is given by

$$
\mu\left(r_{+}\right)=\frac{r_{+}^{d-9}}{(d-7)(d-5)(d-3)}\left(\frac{\gamma^{2} c_{4}}{(d-9)}+\frac{1}{192(d-8)(d-6)}\left[\frac{Q^{2} r_{+}^{2(8-d)}}{(d-7)(d-4)}-\frac{P^{2}}{4(d-9)}\right]\right) .
$$

Figure 3 contains the lapse functions of dyonic black branes in quartic Lovelock theory with horizons given by the product of $m$ two-spheres times $\mathbb{R}^{q}$.

\section{CONCLUSIONS AND FURTHER COMMENTS}

In this paper we have shown that Lovelock theories with a single Lovelock term ${ }^{3}$ of order $n$ admit cylindrical,

\footnotetext{
${ }^{3}$ Lovelock theories with a single Lovelock term also admit for black holes with nonminimally coupled scalar hairs [37].
}

homogeneous charged black strings in the presence of a ( $p-1)$-form, only when $p=n$. In the simplest case, one can consider general relativity $(n=1)$ coupled to a massless scalar field $(p-1=0)$ and construct branelike solutions by just adding flat directions. No-hair theorems in this case forbid the existence of black objects with a nonvanishing scalar field. We have explicitly shown that the next simpler case, i.e., Gauss-Bonnet-Maxwell theory, does admit charged black strings and black branes which are homogeneous on the extended directions. We have extended these results and constructed new explicit 


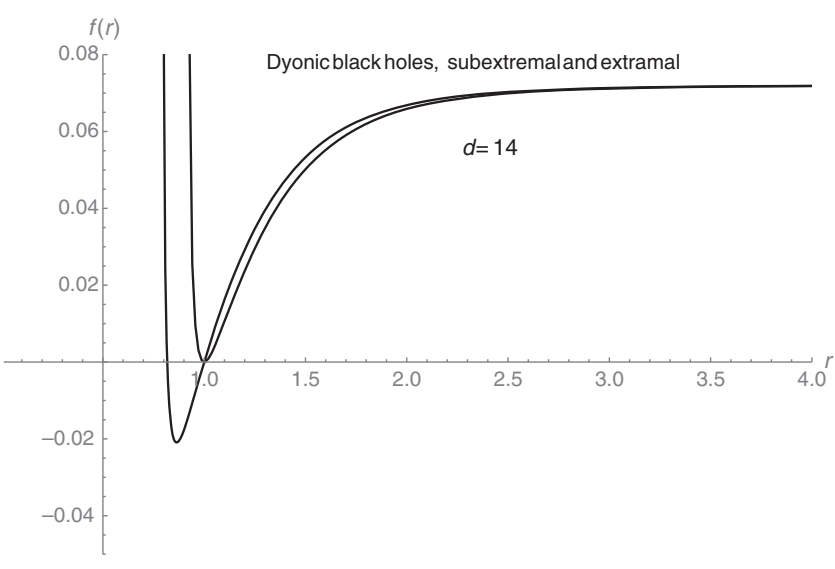

FIG. 3. Dyonic solution in quartic Lovelock theory in dimension $d=14$. The event horizon is given by a product of six twospheres and we have chosen $c_{4}=1, P=1$ and $r_{+}=1$. Two different values for the electric charge are presented which correspond to a subextremal value that leads to a spacetime with an event and Cauchy horizon and also the extremal case for which the horizons degenerate and the solution has vanishing temperature.

solutions, for the cubic Lovelock theory supported by a Kalb-Ramond field as well as for the quartic Lovelock theory with a four-form field strength.

The new black brane solutions we have constructed here have a horizon geometry that is given by the product of spheres times IR ${ }^{q}$. It is well known that in general relativity in vacuum the usual sphere of Schwarzschild-Tangherlini solution [38], hereafter the base manifold, can be replaced by an arbitrary Euclidean Einstein manifold [39]. In Einstein-Gauss-Bonnet theory in vacuum, it was realized by Dotti and Gleiser [40] that for generic values of the Gauss-Bonnet coupling, an Einstein base manifold must also fulfill a quadratic tensor constraint on the curvature that admits a simple expression when written in terms of the Weyl tensor. Some explicit examples of horizons were given in [41] as well as the expression for the finite quasilocal mass. In Refs. [42-44], it was shown that for generic values of the couplings, the Einstein restriction on the base manifold is a necessary condition and that for the particular relation that leads to a theory with a single maximally symmetric solution, the restrictions are weakened and the set of possible causal structures is enlarged to admit as well wormhole solutions in vacuum [45]. This analysis was extended to Lovelock theories in the ChernSimons case [46] in Ref. [47] obtaining similar results even with time dependence. Also products of Thurston geometries may appear as models for the horizon in Lovelock theories with a unique maximally symmetric solution [48]. For arbitrary values of the Lovelock couplings a thorough analysis was provided in Ref. [49], where it was shown that the base manifold has to fulfill a hierarchy of Euclidean
Lovelock equations. A similar result was recently obtained for warped black strings in [50].

Of particular relevance for the present work is Ref. [51], where new static solutions in general relativity coupled to $p$-forms were presented. Here we have used the same ansatz for the $p$-forms in the electric and magnetic cases, where the components are distributed on a symmetric fashion along the base manifold. These results were extended to the Einstein-Gauss-Bonnet theory in [52]. In the latter references the authors also consider base manifolds that are products of Einstein-Kähler spaces, with a $p$-form field strength that is proportional to exterior products of the Kähler forms of each manifold. An exhaustive analysis of the solutions supported by a $p$-form in general relativity in the Robinson-Trautman family was provided in [53,54]. All these solutions, as the ones presented in Sec. IV of this paper, have an asymptotic behavior that matches the transverse section of the metric (49). These metrics have a singularity at $r=0$, which for black hole spacetimes is surrounded by an event horizon.

The stability of homogeneous black strings and black branes is easier to study than that of the nonhomogeneous objects. ${ }^{4}$ Since the solutions are symmetric under translations along the extended directions, one can construct plane waves with a definite momentum along such directions. In general relativity [3], Gauss-Bonnet [28] and cubic Lovelock theories [29] there is a perturbative instability, a Gregory-Laflamme instability, that it is triggered by modes with wavelength above a critical value, and it is therefore a long wavelength instability, sharing some properties of instabilities of other physical systems [55]. It would be interesting to explore the stability of the solutions here constructed, in particular that of the extremal dyonic case in quartic Lovelock theory.

\section{ACKNOWLEDGMENTS}

M.L. and A. V. appreciate the support of CONICYT Fellowships No. 21141229 and No. 21151067, respectively. J. O. thanks Gastón Giribet for enlightening discussions. This work was also funded by CONICYT Grant No. DPI20140053 (J. O. and A.G.) and FONDECYT Grants No. 1150246 and No. 1181047.

\footnotetext{
${ }^{4}$ Even though the Cauchy problem for theories containing a unique Lovelock term could be ill posed around a generic background, Refs. [28,29] show that the linearized problem can be explored in a systematic fashion, providing a well-defined result. As it occurs in vacuum for spherically symmetric black holes, we believe that the configurations we have constructed in this work will appear as the high-curvature regime of charged black string solutions including also the Einstein-Hilbert term. Those solutions might be constructed numerically and in such a setup one could hope to study the nonlinear evolution of the perturbation.
} 
[1] R. Emparan and H.S. Reall, A Rotating Black Ring Solution in Five Dimensions, Phys. Rev. Lett. 88, 101101 (2002).

[2] R. C. Myers and M. J. Perry, Black holes in higher dimensional space-times, Ann. Phys. (N.Y.) 172, 304 (1986).

[3] R. Gregory and R. Laflamme, Black Strings and p-Branes are Unstable, Phys. Rev. Lett. 70, 2837 (1993).

[4] R. Gregory and R. Laflamme, The instability of charged black strings and p-branes, Nucl. Phys. B428, 399 (1994).

[5] R. Gregory and R. Laflamme, Evidence for stability of extremal black p-branes, Phys. Rev. D 51, R305 (1995).

[6] M. W. Choptuik, L. Lehner, I. Olabarrieta, R. Petryk, F. Pretorius, and H. Villegas, Towards the final fate of an unstable black string, Phys. Rev. D 68, 044001 (2003).

[7] D. Garfinkle, L. Lehner, and F. Pretorius, A numerical examination of an evolving black string horizon, Phys. Rev. D 71, 064009 (2005).

[8] L. Lehner and F. Pretorius, Black Strings, Low Viscosity Fluids, and Violation of Cosmic Censorship, Phys. Rev. Lett. 105, 101102 (2010).

[9] P. Figueras, M. Kunesch, and S. Tunyasuvunakool, End Point of Black Ring Instabilities and the Weak Cosmic Censorship Conjecture, Phys. Rev. Lett. 116, 071102 (2016).

[10] P. Figueras, M. Kunesch, L. Lehner, and S. Tunyasuvunakool, End Point of the Ultraspinning Instability and Violation of Cosmic Censorship, Phys. Rev. Lett. 118, 151103 (2017).

[11] D. Lovelock, The Einstein tensor and its generalizations, J. Math. Phys. (N.Y.) 12, 498 (1971).

[12] R. Zegers, Birkhoff's theorem in Lovelock gravity, J. Math. Phys. (N.Y.) 46, 072502 (2005).

[13] S. Deser and J. Franklin, Birkhoff for Lovelock redux, Classical Quantum Gravity 22, L103 (2005).

[14] D. G. Boulware and S. Deser, String Generated Gravity Models, Phys. Rev. Lett. 55, 2656 (1985).

[15] J. T. Wheeler, Symmetric solutions to the Gauss-Bonnet extended Einstein equations, Nucl. Phys. B268, 737 (1986).

[16] J. T. Wheeler, Symmetric solutions to the maximally GaussBonnet extended Einstein equations, Nucl. Phys. B273, 732 (1986).

[17] C. Garraffo and G. Giribet, The Lovelock black holes, Mod. Phys. Lett. A 23, 1801 (2008).

[18] C. Charmousis, Higher order gravity theories and their black hole solutions, Lect. Notes Phys. 769, 299 (2009).

[19] T. Kobayashi and T. Tanaka, Five-dimensional black strings in Einstein-Gauss-Bonnet gravity, Phys. Rev. D 71, 084005 (2005).

[20] Y. Brihaye, T. Delsate, and E. Radu, Einstein-Gauss-Bonnet black strings, J. High Energy Phys. 07 (2010) 022.

[21] B. Kleihaus, J. Kunz, E. Radu, and B. Subagyo, Spinning black strings in five-dimensional Einstein-Gauss-Bonnet gravity, Phys. Lett. B 713, 110 (2012).

[22] J. Crisostomo, R. Troncoso, and J. Zanelli, Black hole scan, Phys. Rev. D 62, 084013 (2000).

[23] R. Aros, R. Troncoso, and J. Zanelli, Black holes with topologically nontrivial AdS asymptotics, Phys. Rev. D 63, 084015 (2001).

[24] R. G. Cai, Gauss-Bonnet black holes in AdS spaces, Phys. Rev. D 65, 084014 (2002); A note on thermodynamics of black holes in Lovelock gravity, Phys. Lett. B 582, 237
(2004); R. G. Cai and N. Ohta, Black holes in pure Lovelock gravities, Phys. Rev. D 74, 064001 (2006).

[25] G. Giribet, J. Oliva, and R. Troncoso, Simple compactifications and black p-branes in Gauss-Bonnet and Lovelock theories, J. High Energy Phys. 05 (2006) 007.

[26] D. Kastor and R. B. Mann, On black strings and branes in Lovelock gravity, J. High Energy Phys. 04 (2006) 048.

[27] A. Cisterna and J. Oliva, Exact black strings and p-branes in general relativity, Classical Quantum Gravity 35, 035012 (2018).

[28] A. Giacomini, J. Oliva, and A. Vera, Black strings in GaussBonnet theory are unstable, Phys. Rev. D 91, 104033 (2015).

[29] A. Giacomini, C. Henriquez-Baez, M. Lagos, J. Oliva, and A. Vera, Instability of black strings in the third-order Lovelock theory, Phys. Rev. D 93, 104005 (2016).

[30] M. Lagos, J. Oliva, and A. Vera, Instability of black $p$-branes in Gauss-Bonnet theory in ten dimensions, The Fourteenth Marcel Grossmann Meeting, 2017, pp. 17391744, https://doi.org/10.1142/9789813226609_0177.

[31] A. I. Janis, E. T. Newman, and J. Winicour, Reality of the Schwarzschild Singularity, Phys. Rev. Lett. 20, 878 (1968).

[32] B. C. Xanthopoulos and T. Zannias, Einstein gravity coupled to a massless scalar field in arbitrary space-time dimensions, Phys. Rev. D 40, 2564 (1989).

[33] S. H. Hendi and M. H. Dehghani, Taub-NUT black holes in third order Lovelock gravity, Phys. Lett. B 666, 116 (2008); M. H. Dehghani, N. Bostani, and S. H. Hendi, Magnetic branes in third order Lovelock-Born-Infeld gravity, Phys. Rev. D 78, 064031 (2008); S. H. Hendi, S. Panahiyan, and H. Mohammadpour, Third order Lovelock black branes in the presence of a nonlinear electromagnetic field, Eur. Phys. J. C 72, 2184 (2012); S. H. Hendi and A. Dehghani, Thermodynamics of third-order Lovelock-AdS black holes in the presence of Born-Infeld type nonlinear electrodynamics, Phys. Rev. D 91, 064045 (2015).

[34] T. Jacobson and R.C. Myers, Black Hole Entropy and Higher Curvature Interactions, Phys. Rev. Lett. 70, 3684 (1993).

[35] K. Copsey and R. Mann, Pathologies in asymptotically Lifshitz spacetimes, J. High Energy Phys. 03 (2011) 039.

[36] M. Barriola and A. Vilenkin, Gravitational Field of a Global Monopole, Phys. Rev. Lett. 63, 341 (1989).

[37] F. Correa and M. Hassaine, Thermodynamics of Lovelock black holes with a nonminimal scalar field, J. High Energy Phys. 02 (2014) 014.

[38] F. R. Tangherlini, Schwarzschild field in $\mathrm{n}$ dimensions and the dimensionality of space problem, Nuovo Cimento 27, 636 (1963).

[39] G. Gibbons and S. A. Hartnoll, A gravitational instability in higher dimensions, Phys. Rev. D 66, 064024 (2002).

[40] G. Dotti and R. J. Gleiser, Obstructions on the horizon geometry from string theory corrections to Einstein gravity, Phys. Lett. B 627, 174 (2005).

[41] H. Maeda, Gauss-Bonnet black holes with nonconstant curvature horizons, Phys. Rev. D 81, 124007 (2010).

[42] G. Dotti, J. Oliva, and R. Troncoso, Exact solutions for the Einstein-Gauss-Bonnet theory in five dimensions: Black holes, wormholes and spacetime horns, Phys. Rev. D 76, 064038 (2007). 
[43] G. Dotti, J. Oliva, and R. Troncoso, Vacuum solutions with nontrivial boundaries for the Einstein-Gauss-Bonnet theory, Int. J. Mod. Phys. A 24, 1690 (2009).

[44] G. Dotti, J. Oliva, and R. Troncoso, Static solutions with nontrivial boundaries for the Einstein-Gauss-Bonnet theory in vacuum, Phys. Rev. D 82, 024002 (2010).

[45] G. Dotti, J. Oliva, and R. Troncoso, Static wormhole solution for higher-dimensional gravity in vacuum, Phys. Rev. D 75, 024002 (2007).

[46] J. Zanelli, Lecture notes on Chern-Simons (super)gravities. Second edition, arXiv:hep-th/0502193.

[47] J. Oliva, All the solutions of the form $M 2$ (warped) $x \Sigma(d-2)$ for Lovelock gravity in vacuum in the Chern-Simons case, J. Math. Phys. (N.Y.) 54, 042501 (2013).

[48] A. Anabalon, F. Canfora, A. Giacomini, and J. Oliva, Black holes with gravitational hair in higher dimensions, Phys. Rev. D 84, 084015 (2011).

[49] S. Ray, Birkhoff's theorem in Lovelock gravity for general base manifolds, Classical Quantum Gravity 32, 195022 (2015).
[50] D. Kastor, S. Ray, and J. Traschen, Lovelock branes, Classical Quantum Gravity 34, 195005 (2017).

[51] Y. Bardoux, M. M. Caldarelli, and C. Charmousis, Shaping black holes with free fields, J. High Energy Phys. 05 (2012) 054.

[52] Y. Bardoux, C. Charmousis, and T. Kolyvaris, Lovelock solutions in the presence of matter sources, Phys. Rev. D 83, 104020 (2011).

[53] M. Ortaggio, J. Podolsky, and M. Zofka, Static and radiating p-form black holes in the higher dimensional Robinson-Trautman class, J. High Energy Phys. 02 (2015) 045.

[54] M. Ortaggio, J. Podolsky, and M. Zofka, Higher dimensional Robinson-Trautman spacetimes sourced by p-forms: Static and radiating black holes, arXiv:1604 .04853 .

[55] V. Cardoso and O. J. C. Dias, Rayleigh-Plateau and Gregory-Laflamme Instabilities of Black Strings, Phys. Rev. Lett. 96, 181601 (2006). 